\title{
Stereotactic body radiation therapy for liver oligo-recurrence and oligo-progression from various tumors
}

\author{
Yu Jin Cha, MD, Mi-Sook Kim, MD, PhD, Won-Il Jang, MD, Young Seok Seo, MD, \\ Chul Koo Cho, MD, PhD, Hyung Jun Yoo, MD, Eun Kyung Paik, MD \\ Department of Radiation Oncology, Korea Institute of Radiological \&t Medical Sciences, Seoul, Korea
}

Purpose: To evaluate the outcomes of stereotactic body radiation therapy (SBRT) for patients with liver oligo-recurrence and oligo-progression from various primary tumors.

Materials and Methods: Between 2002 and 2013, 72 patients with liver oligo-recurrence (oligo-metastasis with a controlled primary tumor) and oligo-progression (contradictory progression of a few sites of disease despite an overall tumor burden response to therapy) underwent SBRT. Of these, 9 and 8 patients with uncontrollable distant metastases and patients immediate loss to follow-up, respectively, were excluded. The total planning target volume was used to select the SBRT dose (median, 48 Gy; range, 30 to 60 Gy, 3-4 fractions). Toxicity was evaluated using the Common Toxicity Criteria for Adverse Events v4.0.

Results: We evaluated 55 patients (77 lesions) treated with SBRT for liver metastases. All patients had controlled primary lesions, and 28 patients had stable lesions at another site (oligo-progression). The most common primary site was the colon (36 patients), followed by the stomach ( 6 patients) and other sites (13 patients). The 2-year local control and progression-free survival rates were $68 \%$ and 22\%, respectively. The 2 - and 5-year overall survival rates were 56\% and 20\%, respectively. The most common adverse events were grade 1-2 fatigue, nausea, and vomiting; no grade $\geq 3$ toxicities were observed. Univariate analysis revealed that oligoprogression associated with poor survival.

Conclusion: SBRT for liver oligo-recurrence and oligo-progression appears safe, with similar local control rates. For liver oligoprogression, criteria are needed to select patients in whom improved overall survival can be expected through SBRT.

Keywords: Liver metastases, Oligo-recurrence, Oligo-progression, Stereotactic radiation therapy

\section{Introduction}

Liver metastasis most commonly occurs secondary to colorectal cancer (CRC), followed by breast and gastrointestinal cancers [1]. Although hematogenous metastasis to the liver from various malignancies is often considered incurable, curative local therapy can be attempted for single or oligo-metastasis to the liver. The first-line choice for curative treatment of liver metastases originating from CRC is surgical resection, which has been reported to provide 5-year survival rates of 37\%$58 \%[2,3]$. These data suggest that a subset of patients with limited liver metastases could experience extended long-term survival by local operation [4]. However, $>80 \%$ of patients with liver metastases are unsuitable for surgery, which is reserved for a select group of patients with appropriate medical conditions and reserved hepatic volume [5]. Transcatheter arterial chemoembolization and radiofrequency ablation are considered alternative local treatments for managing liver

Received 10 January 2017, Revised 24 April 2017, Accepted 02 June 2017.

Correspondence: Mi-Sook Kim, MD, PhD, Department of Radiation Oncology, Korea Institute of Radiological \& Medical Sciences, 75 Nowon-ro, Nowon-gu, Seoul 01812, Korea. Tel: +82-2-970-2484, Fax: +82-2-970-2412, E-mail: mskim@kirams.re.kr

(c) This is an Open Access article distributed under the terms of the Creative Commons Attribution Non-Commercial License (http://creativecommons.org/ licenses/by-nc/4.0/) which permits unrestricted non-commercial use, distribution, and reproduction in any medium, provided the original work is properly cited.

www.e-roj.org 
metastases, although these procedures are limited by the tumor's diameter and location.

Radiotherapy has traditionally been considered a palliative treatment for primary or metastatic liver tumors, as it is limited by the low tolerance of the liver and the potential for radiation-induced liver disease. However, advances in stereotactic body radiotherapy (SBRT) have made it possible to administer highly focused treatments of high-dose radiation in one or a few fractions, while sparing the normal surrounding tissues with a rapid dose fall-off. In addition, recent evidence suggests that SBRT indirectly leads to tumor cell death by compromising the intra-tumor microenvironment and by the abscopal effect, which is induced by the subsequent immune boost $[6,7]$.

The term 'oligo-metastases' was first introduced in 1995 to describe an intermediate state of cancer spread between localized and widespread disease [8]. Niibe and Hayakawa [9] suggested a further conceptual refinement using the concept of 'oligo-recurrence' as oligo-metastasis with a controlled primary tumor. Patients with oligo-recurrence typically have a better prognosis compared to patients with oligo-metastasis [10]. Recently, the emerging concept of 'oligo-progression' defined as the contradictory progression of one or a few sites of disease despite an overall tumor burden response to therapy (that is, with the exception of a few growing tumors, the majority of other tumors are not progressing) was introduced [11]. With the recent advances in chemotherapy, some patients with disseminated disease can now be converted to having an oligo-progression status. Therefore, even among tumors of the same pathology, these newly determined subclasses among distant metastases demonstrate remarkable heterogeneity, not only in the treatment response but also in their phenotypes and ability for metastatic spread. Fortunately, in these patients, SBRT can provide local control of the progressing sites of disease so that (1) the current systemic treatment that is controlling the disease can be maintained and (2) the initiation of additional aggressive chemotherapy regimens can be limited or delayed. Although several recent publications have reported the effectiveness of aggressive local modalities in select patients with systemic disease, there is only limited evidence supporting an overall survival (OS) gain [12,13]. Moreover, the suitable candidates for SBRT among patients with oligometastases should be determined.

In particular, while many previous studies have revealed that SBRT is an effective local modality for liver metastases, especially for oligo-metastases and oligo-recurrence, it remains unclear whether this approach is also effective for case of oligo-progression. With this in mind, the present study aimed to evaluate the clinical outcomes of SBRT for liver oligorecurrence and liver oligo-progression among patients with various primary cancers.

\section{Materials and Methods}

\section{Study design and patients}

This study retrospectively evaluated all consecutive 72 patients with liver oligo-recurrence and oligo-progression who underwent SBRT at the Korea Cancer Center Hospital between January 2002 and August 2013. All patients had available medical records and radiographic examination data. Eight patients were excluded because of immediate loss of follow-up. Moreover, 9 patients with uncontrollable distant metastases were also excluded from the analyses. Among the remaining 55 patients, 27 patients were classified to have liver oligo-recurrence, defined as $<5$ lesions in the liver only and a controlled primary tumor, while 28 patients were classified as having liver oligo-progression, defined as $<5$ lesions in the liver and an inactive extra-hepatic lesion located in the lung para-aortic lymph nodes, or other locations (Table 1). Inactive lesions were defined as lesions stable for at least 3 months on follow-up radiological examinations, regardless of the specific systemic therapy regimen used. In all patients, their primary site was controlled by surgery/radiation therapy.

All patients underwent chemotherapy before or after treatment of the primary site. After the diagnosis of liver recurrence, 39 (71\%) of the 55 patients received additional chemotherapy. Finally, 77 liver lesions in 55 patients were analyzed (Tables 2 and 3). This study was approved by the Institutional Review Board (No. K-1703-002-085).

\section{SBRT procedures}

SBRT was performed using either a CyberKnife (Accuray Inc., Sunnyvale, CA, USA) or a RapidArc (Varian Medical Systems, Palo Alto, CA, USA). All patients were immobilized in the supine position, with abdominal compression that limited diaphragmatic excursion and any respiration-related tumor motion [14]. Computed tomography (CT) simulation was performed using slow, free-breathing CT images (slice thickness, $2.5 \mathrm{~mm}$ ). Magnetic resonance imaging and/or positron emission tomography/CT were also used to provide reference images to further delineate the tumor volume. The gross tumor volume included the tumor margins seen on free-breathing and slow CT images to compensate for target movement due to breathing. The clinical target volume was 
Table 1. Clinicodemographic characteristics of the study patients

\begin{tabular}{|c|c|}
\hline Characteristic & Value \\
\hline \multicolumn{2}{|l|}{ Patient characteristics $(n=55)$} \\
\hline Age (yr) & $63(42-86)$ \\
\hline \multicolumn{2}{|l|}{ Sex } \\
\hline Male & $30(55)$ \\
\hline Female & $25(45)$ \\
\hline \multicolumn{2}{|l|}{ Primary tumor } \\
\hline Colorectal cancer & $36(65)$ \\
\hline Stomach cancer & $6(11)$ \\
\hline Cervical cancer & $4(7)$ \\
\hline Breast cancer & $2(4)$ \\
\hline Pancreatic cancer & $2(4)$ \\
\hline Others ${ }^{\text {a) }}$ & $5(9)$ \\
\hline \multicolumn{2}{|l|}{ Histology } \\
\hline Adenocarcinoma & $45(82)$ \\
\hline Squamous cell carcinoma & $4(7)$ \\
\hline Others & $6(11)$ \\
\hline \multicolumn{2}{|l|}{ Extra-hepatic disease } \\
\hline Yes (inactive) & $28(51)$ \\
\hline $\mathrm{No}^{\mathrm{c}}$ & $27(49)$ \\
\hline \multicolumn{2}{|c|}{ Extra-hepatic site (for oligo-progression, $n=28$ ) } \\
\hline Lung & $8(29)$ \\
\hline Para-aortic lymph nodes & $12(42)$ \\
\hline Others ${ }^{\mathrm{d})}$ & $8(29)$ \\
\hline \multicolumn{2}{|l|}{ Intra-hepatic recurrent tumors } \\
\hline 1 & $38(69)$ \\
\hline 2 & $14(25)$ \\
\hline $3-5$ & $3(6)$ \\
\hline \multicolumn{2}{|c|}{ Interval from liver oligo-recurrence diagnosis to } \\
\hline SBRT (mo) & $2(0-22)$ \\
\hline \multicolumn{2}{|c|}{ Chemotherapy for liver recurrence } \\
\hline Yes & $39(71)$ \\
\hline No & $16(29)$ \\
\hline \multicolumn{2}{|l|}{ Tumor characteristics $(n=77)$} \\
\hline \multicolumn{2}{|l|}{ Tumor volume $(\mathrm{mL})$} \\
\hline$\leq 10$ & $37(48)$ \\
\hline$>10$ and $\leq 100$ & $26(34)$ \\
\hline$>100$ & $14(18)$ \\
\hline
\end{tabular}

Values are presented as number (\%) or median (range).

SBRT, stereotactic body radiation therapy.

${ }^{a}$ Ampulla of Vater cancer, primary unknown cancer, gastrointestinal stromal tumor, or chordoma. be Leiomyosarcoma, invasive ductal carcinoma, gastrointestinal stromal tumor, or chordoma. ${ }^{\text {c} A b s e n c e ~ o f ~}$

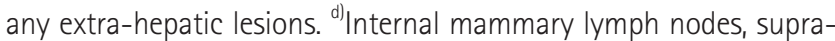
clavicular lymph nodes, or mesentery.

defined using margins of 1-3 $\mathrm{mm}$ around the gross tumor volume, and was expanded by a 3-5 $\mathrm{mm}$ margin to create the planning target volume (PTV). However, the PTV margins were modified in some patients because of the presence of normal tissue, such as the spinal cord, stomach, or intestines.

\section{Treatment characteristics}

The patients' prescribed doses, doses per fraction, and number of fractions were determined based on their tumor size, tumor location, and organs at risk. All patients received 3-4 fractions, with a median total dose of $48 \mathrm{~Gy}$ (range, 30 to $60 \mathrm{~Gy}$ ). The median biologically effective dose was $113 \mathrm{~Gy}_{10}$ (range, 58 to $\left.180 \mathrm{~Gy}_{10}\right)$, when $\alpha / \beta$ was assumed to be $10 \mathrm{~Gy}[15]$.

\section{Follow-up after SBRT and toxicity assessments}

The clinical responses were evaluated using abdominal CT at 1-3 months, based on the Response Evaluation and Criteria in Solid Tumors [16]. The study outcomes were defined as the local control (LC), progression-free survival (PFS), and OS rates. LC was defined as complete or partial responses or stable disease. PFS was defined as the time from SBRT treatment to the first instance of disease progression or the last followup. OS was defined as the time from the liver oligo-recurrence diagnosis to the date of death; patients were censored if they were alive at the last follow-up.

Acute and late toxicities were defined based on laboratory findings and symptoms that developed at $<3$ or $\geq 3$ months after the SBRT, respectively. Toxicities were categorized using the Common Terminology Criteria for Adverse Events ver. 4.0.

\section{Statistical analysis}

The Kaplan-Meier method was used to estimate the survival rates, which were compared using the log-rank test. We also performed univariate analyses using a Cox proportional hazards regression model. Factors with a p-value of $<0.05$ were considered statistically significant. SPSS ver. 23 (IBM SPSS, Armonk, NY, USA) was used for all statistical analyses.

\section{Results}

\section{Patient and tumor characteristics}

The 55 patients included 30 men and 25 women, with a median age of 63 years (range, 42 to 86 years) (Table 1). The most common primary tumor was CRC (36 patients, 65\%), followed by stomach ( 6 patients, 11\%), cervical (4 patients, 7\%), breast (2 patients, 4\%), and other cancers (7 patients, 13\%). All 55 patients and 77 lesions were assessed for LC, PFS, and OS. The median interval from the liver oligo-recurrence diagnosis to SBRT was 2 months (range, 0 to 22 months). All 28 patients with oligo-progression had a single extra-hepatic inactive lesion. The most common location of the extra-hepatic inactive lesions was the para-aortic lymph nodes (12/28, 43\%), followed by the lungs $(8 / 28,29 \%)$. The median PTV of the liver recurrences was $11 \mathrm{~mL}$ (range, 0.3 to $721 \mathrm{~mL}$ ).

\section{Tumor response and local control}

The median follow-up duration for assessing LC after SBRT 
A

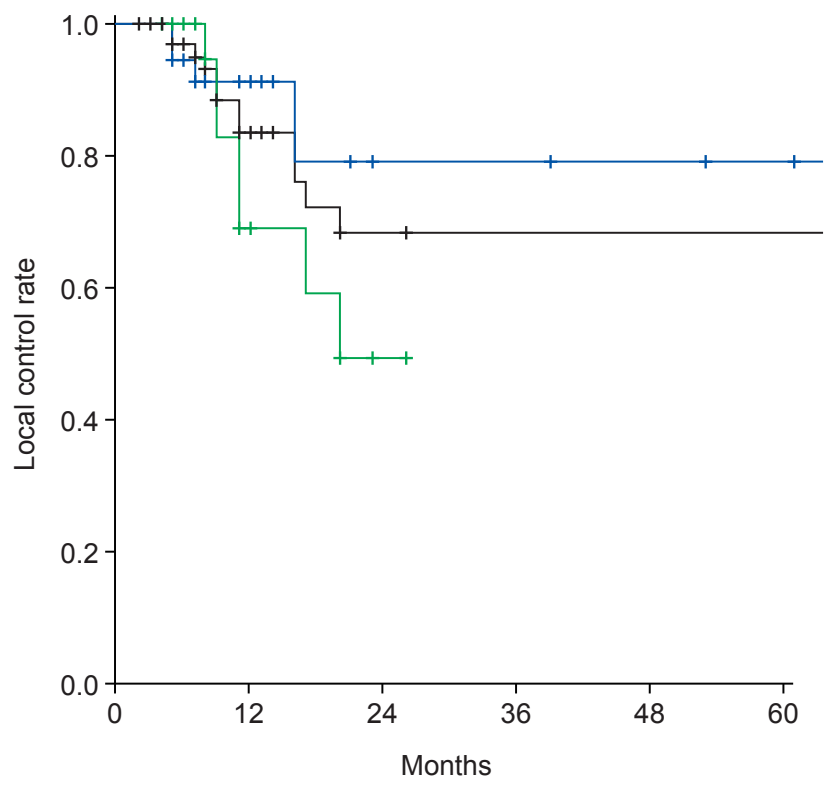

C

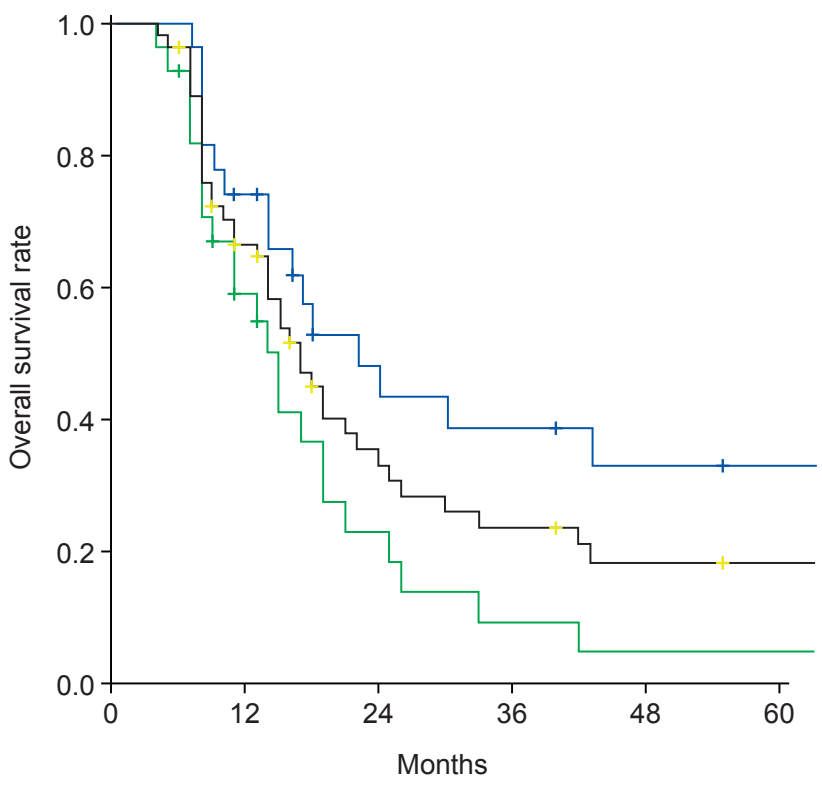

B

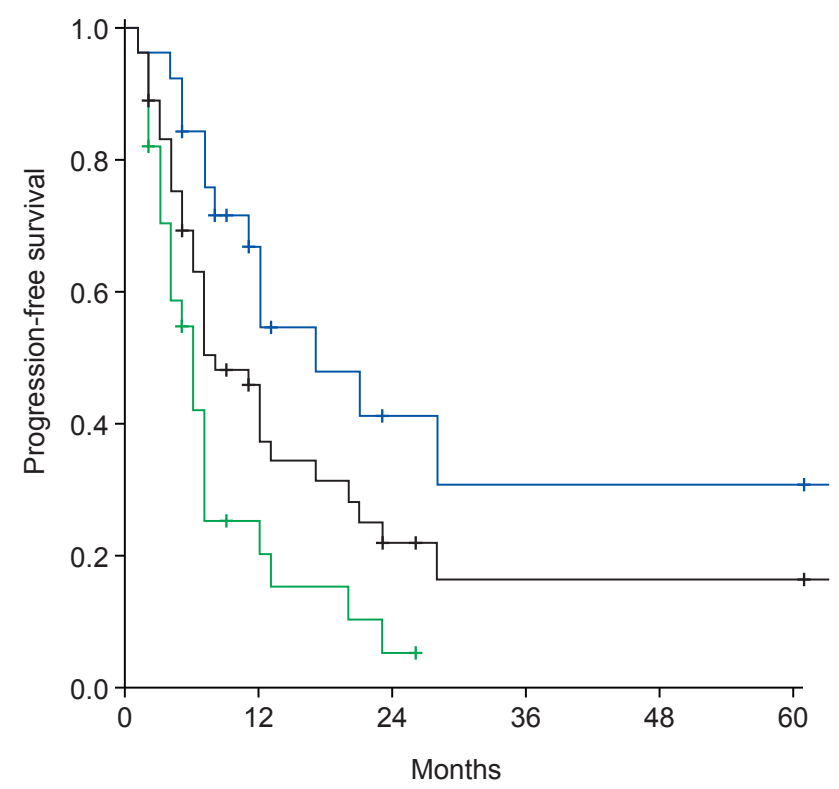

Fig. 1. Stereotactic body radiation therapy outcomes. (A) Local control rate. (B) Progression-free survival. (C) Overall survival. The bold black lines represent the overall rates. The blue and green lines represent the rates for patients with oligo-recurrence and oligo-progression, respectively.

was 9 months (range, 2 to 83 months). The overall 1 - and 2-year LC rates were $83 \%$ and $68 \%$, respectively (Fig. 1). In the univariate analyses, better LC was significantly associated with a biologically effective dose of $>110 \mathrm{~Gy}_{10}(p=0.021)$ and a PTV of $<10 \mathrm{~mL}$ ( $p=0.001$ ) (Table 2). Specifically, 1-year LC rates of $95 \%, 86 \%$, and $44 \%$ were seen among tumors $<10 \mathrm{~mL},>10$ $\mathrm{mL}$ but $<100 \mathrm{~mL}$, and $>100 \mathrm{~mL}$, respectively. The 2-year $\mathrm{LC}$ rate in patients with CRC was $79 \%$, as compared to $21 \%$ in those with other primary tumors (Table 2). CRC tumors showed a tendency to be irradiated with a marginally higher dose ( $p$ $=0.073$, Mann-Whitney $\mathrm{U}$ test) than other types of tumors, despite no differences in size ( $p=0.231$, Mann-Whitney $U$ test) and the use of chemotherapy.

\section{Progression-free and overall survivals}

The median follow-up duration for assessing survival after the liver recurrence diagnosis was 24 months (range, 4 to 120 months). Thirty-nine patients (71\%) had died at the time of our analysis. The 1- and 2-year PFS rates were $46 \%$ and $22 \%$, respectively. The univariate analyses revealed that PFS was 
Table 2. Univariate analyses of factors predicting LC

\begin{tabular}{|c|c|c|c|c|}
\hline Characteristic & No. of patients & $1-y r$ LC (\%) & 2-yr LC (\%) & $p$-value \\
\hline \multicolumn{5}{|l|}{ Age (yr) } \\
\hline$\leq 63$ & 38 & 80 & 73 & \multirow[t]{2}{*}{0.751} \\
\hline$>63$ & 39 & 84 & 63 & \\
\hline \multicolumn{5}{|l|}{ Sex } \\
\hline Male & 44 & 95 & 71 & \multirow[t]{2}{*}{0.141} \\
\hline Female & 33 & 70 & 63 & \\
\hline \multicolumn{5}{|l|}{ Primary cancer } \\
\hline CRC & 57 & 83 & 79 & \multirow[t]{2}{*}{0.104} \\
\hline Others ${ }^{\text {a) }}$ & 20 & 82 & 21 & \\
\hline \multicolumn{5}{|l|}{$\mathrm{BED}\left(\mathrm{Gy}_{10}\right)$} \\
\hline$>110$ & 49 & 92 & 84 & \multirow[t]{2}{*}{0.021} \\
\hline$\leq 110$ & 28 & 68 & 50 & \\
\hline \multicolumn{5}{|c|}{ Extra-hepatic inactive lesions ${ }^{\text {b) }}$} \\
\hline Yes & 39 & 69 & 49 & \multirow[t]{2}{*}{0.118} \\
\hline No & 38 & 91 & 79 & \\
\hline \multicolumn{5}{|l|}{ Chemotherapy } \\
\hline Yes & 57 & 83 & 61 & \multirow[t]{2}{*}{0.677} \\
\hline No & 20 & 83 & 83 & \\
\hline \multicolumn{5}{|l|}{ Planning target volume (mL) } \\
\hline$\leq 10$ & 37 & 95 & 95 & \multirow[t]{2}{*}{0.001} \\
\hline$>10$ & 40 & 70 & 42 & \\
\hline
\end{tabular}

LC, local control; $C R C$, colorectal cancer; BED, biologically effective dose.

${ }^{a}$ All types of primary tumors shown in Table 1, except CRC. ${ }^{b}$ Presence of an extra-hepatic gross tumor at the time of diagnosis of the treatment target intra-hepatic tumor.

only predicted by extra-hepatic disease $(p=0.002)$. Among all patients, the 2- and 5-year OS rates were 56\% and 20\%, respectively, with a median survival of 40 months (Fig. 1). In the univariate analyses, OS was significantly predicted by the oligo-progression ( $p=0.007)$ and the cumulative PTV $(p=0.05)$ (Table 3).

\section{Patterns of failure}

During the follow-up, we identified disease progression in 38 of the 55 patients (69\%). Extra-hepatic recurrence was the most common type of recurrence, and involved the lungs (14 patients), bone (7 patients), lymph nodes (7 patients), and peritoneum (1 patient). Local recurrence occurred in 11 patients. The patterns of failure in patients with oligorecurrence and oligo-progression are shown in Fig. 2. The frequency of extra-hepatic recurrence was higher in patients with oligo-progression (21/28 patients, 75\%) than in those with oligo-recurrence (8/27 patients, 30\%).

\section{Toxicity}

The treatment was well tolerated by all patients. Grade 1-2 nausea, vomiting, and fatigue were the most common toxicities (15 patients, 27\%) and were corrected using routine care. Grade 2 ascites was observed in 2 patients (4\%). We did not detect any cases of grade 3 or higher adverse acute events
Table 3. Univariate analyses of factors predicting PFS and OS

\begin{tabular}{lcc}
\hline & \multicolumn{2}{c}{$p$-value } \\
\cline { 2 - 3 } & PFS & OS \\
\hline Age ( $\leq 63$ vs. $>63$ yr) & 0.619 & 0.553 \\
Sex (male vs. female) & 0.034 & 0.956 \\
Primary cancer (CRC vs. other) & 0.544 & 0.072 \\
BED (>110 vs. $\leq 110$ Gy $_{10}$ ) & 0.588 & 0.062 \\
Disease type & & \\
$\quad$ (oligo-progression vs. oligo-recurrence) & 0.002 & 0.007 \\
Chemotherapy (yes vs. no) & 0.942 & 0.571 \\
cPTV ( $\leq 50$ vs. $>50$ mL) & 0.057 & 0.050 \\
SBRT interval a) ( $\leq 12$ vs. >12 mo) & 0.019 & 0.271 \\
Total target number (1 vs. 2-5) & 0.244 & 0.360 \\
\hline
\end{tabular}

PFS, progression-free survival; OS, overall survivals; $C R C$, colorectal cancer; BED, biologically effective dose; cPTV, cumulative planning target volume; SBRT, stereotactic body radiation therapy.

a) Time from the liver oligo-recurrence diagnosis to the SBRT treatment.

or treatment-related deaths and there were no reports of any treatment-related adverse events at 3 months after the treatment.

\section{Discussion and Conclusion}

Many previous studies have reported that SBRT provides promising outcomes and favorable LC rates of $>90 \%$ for liver 
A
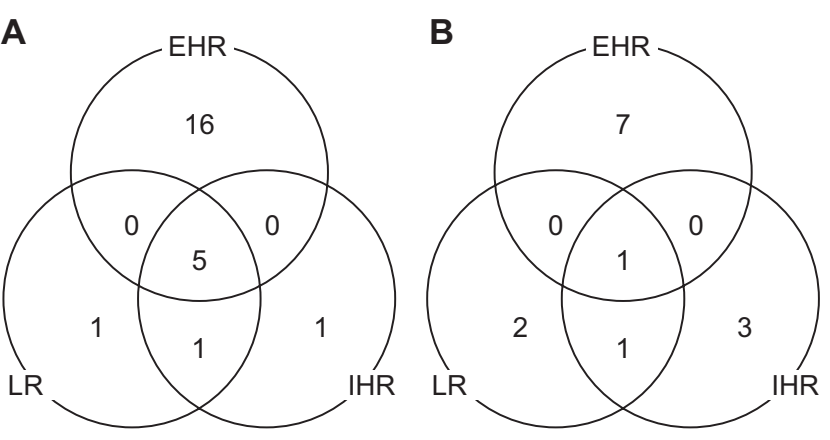

Fig. 2. Failure patterns in patients with oligo-recurrence and oligo-progression. (A) Failure patients among patients with no extra-hepatic lesion (oligo-recurrence) treated by SBRT (14/27 patients). (B) Failure patients among the patients with an extrahepatic (oligo-progression) treated by SBRT (24/28 patients). $S B R T$, stereotactic body radiation therapy; $L R$, local recurrence (recurrence within the field that received SBRT); IHR, intra-hepatic recurrence (recurrence outside the field that received SBRT, but not extra-hepatic recurrence); EHR, extra-hepatic recurrence (recurrence outside the liver, including progression of extrahepatic inactive lesions present during SBRT).

metastases, especially in small-size tumors. Rusthoven et al. [17] performed SBRT (3 fractions, 36-60 Gy) for 63 metastatic liver lesions and reported LC rates of 95\% at 1 year and 92\% at 2 years, with no cases of radiation-induced liver disease. Van der Pool et al. [18] reported LC rates of 100\% at 1 year and $74 \%$ at 2 years using $30-37.5 \mathrm{~Gy}$ in 3 fractions for 20 patients. On the other hand, Lee et al. [19] reported a 1-year LC rate of only $71 \%$ among 68 metastatic liver lesions, likely because they included relatively large tumors (median volume, 75.9 $\mathrm{mL}$; range, 1.2 to $3,090 \mathrm{~mL}$ ). In the present study, we observed 1 - and 2-year LC rates of $83 \%$ and $68 \%$, respectively (median volume, $11 \mathrm{~mL}$; range, 0.3 to $721 \mathrm{~mL}$ ).

The $L C$ rate usually correlates with the tumor size when a curative SBRT dose is delivered to the tumor site. Several studies have revealed that the $L C$ rate is influenced by the target volume, with Lee et al. [19] reporting that better LC was achieved in smaller tumors. Yamashita et al. [20] reported that the $L C$ rate was significantly higher in cases of SBRT for liver metastatic tumors $<30 \mathrm{~mm}$ in diameter. In the present study, we observed that an LC rate of $>95 \%$ was achieved for tumors that had a PTV of $<10 \mathrm{~mL}$. This result is similar to the PTV cutoff used to predict high LC rates in other previous studies. However, several reports have reported that the LC effect is insufficient in relatively bulky liver metastases. Andratschke et al. [21] reported a 1-year LC rate of 74.7\% for 91 liver metastases with a median PTV of $123 \mathrm{~mL}$ treated with 3-5 fractions with 5-12.5 Gy/fraction. Berber et al. [22] reported a 62\% 1-year LC rate in 363 liver metastases with a median PTV of $138 \mathrm{~mL}$ and a total dose of $37.5 \pm 8.2 \mathrm{~Gy}$ in their multicenter database analysis. Klement et al. [23] reported a 2-year LC rate of 58\% in patients receiving chemotherapy before SBRT, using a multi-center database of 452 SBRT treatments with a median PTV of $70.2 \mathrm{~mL}$. Our study showed a 1-year LC rate of $86 \%$ in PTV $>10 \mathrm{~mL}$ but $<100 \mathrm{~mL}$ in size. Although a high LC was observed in tumors $<10 \mathrm{~mL}$, a relatively good $\mathrm{LC}$ might hence still be expected in tumors $<100 \mathrm{~mL}$. On the other hand, the 1 -year $\mathrm{LC}$ rate in tumors $>100 \mathrm{~mL}$ was only $44 \%$. Therefore, further studies are needed to determine the most appropriate treatment strategy to improve the $L C$ rate, including multimodality approaches, even for bulky tumors.

In general, the tumor volume and radiation dose significantly influence the LC after SBRT, although some reports have moreover suggested differences in the treatment outcomes based on the primary histology, with a poorer LC observed in cases of CRC histology. In Ahmed et al's report [24], the multigene expression index for tumor radio-sensitivity showed a poorer radio-response in CRC liver metastases than in other pathologies. This finding was well validated by an independent cohort (100\% LC in non-CRC vs. 79\% LC in CRC at 1 year), despite the validation cohort being small [24]. Moreover, several studies have reported that the $\mathrm{LC}$ rates for oligo-recurrence from CRC were worse than those from other primary tumors [25-27]. However, our results revealed that the LC was not statistically different for CRC oligo-recurrence compared to that from other primary tumors. We speculate that this finding may be because CRC metastases associated with a higher SBRT dose and smaller tumor size ( $p=0.073$ and $p=0.231$, respectively) were compared to metastases from other pathologies. Nevertheless, there are also several reports of an encouraging LC rate of SBRT for CRC oligometastases. Especially, Takeda et al. [28] reported a 2-year LC rate of 100\% with a total dose of 50-60 Gy (5 fractions) for CRC oligo-recurrence in the liver and lung. Interestingly, Kinj et al. [29] reported a lower LC for rectal lesions compared to colon cancer after SBRT for lung oligo-metastases. Moreover, they suggested that rectal lesions may have a more aggressive biological behavior, as suggested by the proportion of KRASmutated tumors. Therefore, even though the LC rate of SBRT for CRC oligo-recurrence may be poorer than that for other tumors, further evaluations of the biological factors responsible for this finding, both through genomic mutational profile analyses and clinical trials, are necessary to clarify this issue. 


\section{Yu Jin Cha, et al}

Oligo-progression refers to the progress of a few metastases while all other metastases are stable or responding to systemic therapy. SBRT for progression of tumors is expected to delay the need for additional, aggressive chemotherapy. Therefore, active local treatment for oligo-progression is expected to improve the quality of life of patients by improving PFS and OS [11]. Developments in diagnostic technology have enabled the detection of all sites of metastatic disease for patients with five or fewer lesions and the identification of metastases that progress despite an otherwise positive treatment response to systemic treatments. Among the treatment options for oligo-progression, the role of local therapy, such as SBRT, is to eradicate the growing metastases and delay the need to change systemic therapy, which may theoretically result in prolonged PFS and OS. However, this hypothesis should be evaluated in various pathologies, because almost all published studies supporting this theory are limited by their retrospective nature or are related to non-small cell lung cancer oligoprogression. In our results, the LC rates among patients with oligo-recurrence and oligo-progression did not significantly differ $(p=0.118)$. Conversely, the OS and PFS differed between these groups, with poorer OS and PFS in patients with oligoprogression compared to oligo-recurrence, as expected. Furthermore, in our results, despite the differences in several factors such as the histology, effectiveness of systemic therapy, and cancer biology between the two groups, the LC achieved by SBRT was similar. However, the poorer PFS rate in the oligoprogression group indicate the limited long-term survival of this group. Therefore, our data are limited for confirming the role of SBRT in patients with oligo-progression, and we consider that more specific criteria for SBRT are necessary in this patient group in order to translate the higher LC of limited progression to a higher OS. A prospective trial may be needed to address the clinical benefits of using SBRT for oligo-metastases, including both oligo-recurrence and oligoprogression.

In conclusion, SBRT was found to be safe for patients with both liver oligo-recurrence and oligo-progression, with similar LC effects. For liver oligo-progression, criteria are needed to select patients in whom OS improvements can be expected through SBRT.

\section{Conflict of Interest}

No potential conflict of interest relevant to this article was reported.

\section{Acknowledgments}

This study was supported by a grant of the Korea Institute of Radiological \& Medical Sciences (KIRAMS), funded by Ministry of Science, ICT and Future Planning, Republic of Korea (Nos. 1711042677,1711045548,1711045553,1711045555/505432017).

\section{References}

1. Kasper HU, Drebber U, Dries V, Dienes HP. Liver metastases: incidence and histogenesis. Z Gastroenterol 2005;43:1149-57.

2. Robertson DJ, Stukel TA, Gottlieb DJ, Sutherland JM, Fisher ES. Survival after hepatic resection of colorectal cancer metastases: a national experience. Cancer 2009;115:752-9.

3. Haddad AJ, Bani Hani M, Pawlik TM, Cunningham SC Colorectal liver metastases. Int J Surg Oncol 2011;2011:285840.

4. Biasco G, Derenzini E, Grazi G, et al. Treatment of hepatic metastases from colorectal cancer: many doubts, some certainties. Cancer Treat Rev 2006;32:214-28.

5. Dawood O, Mahadevan A, Goodman KA. Stereotactic body radiation therapy for liver metastases. Eur J Cancer 2009;45:2947-59.

6. Kim MS, Kim W, Park IH, et al. Radiobiological mechanisms of stereotactic body radiation therapy and stereotactic radiation surgery. Radiat Oncol J 2015;33:265-75.

7. Folkert MR, Timmerman RD. Stereotactic ablative body radiosurgery (SABR) or stereotactic body radiation therapy (SBRT). Adv Drug Deliv Rev 2017;109:3-14.

8. Hellman S, Weichselbaum RR. Oligometastases. J Clin Oncol 1995;13:8-10.

9. Niibe $Y$, Hayakawa K. Oligometastases and oligo-recurrence: the new era of cancer therapy. Jpn J Clin Oncol 2010;40:10711

10. Seo YS, Kim MS, Yoo HJ, Jang WI. Stereotactic body radiotherapy for oligo-recurrence within the nodal area from colorectal cancer. World J Gastroenterol 2014;20:2005-13.

11. Cheung P. Stereotactic body radiotherapy for oligoprogressive cancer. Br J Radiol 2016;89:20160251.

12. Westover KD, lyengar $P$, Sharma $A N$, Timmerman R. SABR for aggressive local therapy of metastatic cancer: a new paradigm for metastatic non-small cell lung cancer. Lung Cancer 2015;89:87-93.

13. Reyes DK, Pienta KJ. The biology and treatment of oligometastatic cancer. Oncotarget 2015;6:8491-524.

14. Eccles CL, Patel R, Simeonov AK, Lockwood G, Haider M, Dawson LA. Comparison of liver tumor motion with and without abdominal compression using cine-magnetic resonance 
imaging. Int J Radiat Oncol Biol Phys 2011;79:602-8.

15. Fowler JF. The linear-quadratic formula and progress in fractionated radiotherapy. Br J Radiol 1989;62:679-94.

16. Therasse $P$, Arbuck SG, Eisenhauer EA, et al. New guidelines to evaluate the response to treatment in solid tumors. European Organization for Research and Treatment of Cancer, National Cancer Institute of the United States, National Cancer Institute of Canada. J Natl Cancer Inst 2000;92:205-16.

17. Rusthoven KE, Kavanagh BD, Cardenes $H$, et al. Multiinstitutional phase I/II trial of stereotactic body radiation therapy for liver metastases. J Clin Oncol 2009;27:1572-8.

18. van der Pool AE, Mendez Romero A, Wunderink W, et al. Stereotactic body radiation therapy for colorectal liver metastases. Br J Surg 2010;97:377-82.

19. Lee MT, Kim JJ, Dinniwell R, et al. Phase I study of individualized stereotactic body radiotherapy of liver metastases. J Clin Oncol 2009;27:1585-91.

20. Yamashita $H$, Onishi $H$, Matsumoto $Y$, et al. Local effect of stereotactic body radiotherapy for primary and metastatic liver tumors in 130 Japanese patients. Radiat Oncol 2014;9:112.

21. Andratschke NH, Nieder C, Heppt F, Molls M, Zimmermann F. Stereotactic radiation therapy for liver metastases: factors affecting local control and survival. Radiat Oncol 2015;10:69.

22. Berber B, Ibarra R, Snyder L, et al. Multicentre results of stereotactic body radiotherapy for secondary liver tumours. HPB (Oxford) 2013;15:851-7.

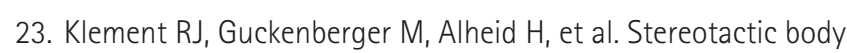

radiotherapy for oligo-metastatic liver disease: influence of pre-treatment chemotherapy and histology on local tumor control. Radiother Oncol 2017;123:227-33.

24. Ahmed KA, Fulp WJ, Berglund AE, et al. Differences between colon cancer primaries and metastases using a molecular assay for tumor radiation sensitivity suggest implications for potential oligometastatic SBRT patient selection. Int J Radiat Oncol Biol Phys 2015;92:837-42.

25. Rule $W$, Timmerman $R$, Tong $L$, et al. Phase I dose-escalation study of stereotactic body radiotherapy in patients with hepatic metastases. Ann Surg Oncol 2011;18:1081-7.

26. Vautravers-Dewas C, Dewas S, Bonodeau F, et al. Imageguided robotic stereotactic body radiation therapy for liver metastases: is there a dose response relationship? Int J Radiat Oncol Biol Phys 2011;81:e39-47.

27. Aitken KL, Tait DM, Nutting CM, Khabra K, Hawkins MA. Riskadapted strategy partial liver irradiation for the treatment of large volume metastatic liver disease. Acta Oncol 2014;53:7026.

28. Takeda A, Sanuki N, Tsurugai Y, Oku Y, Aoki Y. Stereotactic body radiotherapy for patients with oligometastases from colorecta cancer: risk-adapted dose prescription with a maximum dose of 83-100 Gy in five fractions. J Radiat Res 2016;57:400-5.

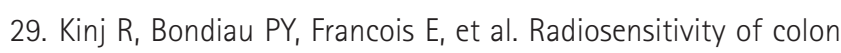
and rectal lung oligometastasis treated with stereotactic ablative radiotherapy. Clin Colorectal Cancer 2016 Aug 31 [Epub]. http://doi.org/10.1016/j.clcc.2016.08.003. 